\title{
Influence of stage of lactation on glutamine metabolism in dairy cows
}

\author{
EK Okine 1, JJ Kennelly 2 \\ 'Alberta agriculture, food and rural development, Edmonton, Alberta, T6H 4P2 ; 'Department of agricultural, \\ food and nutritional science, University of Alberta, Edmonton, Canada
}

In a variety of monogastric species, rapidly dividing cells such as enterocytes derive most of their energy from the oxidation of glucose and glutamine, with glutamine being the major energy source (Hanson and Parsons, 1977, Biochem J, 166, 509-519). However, there is a paucity of information on the fate of nitrogen from glutamine and the relative importance of glucose and glutamine as energy sources in the small intestine of lactating dairy cattle. The metabolism of the small intestine is important because it influences the net presentation of nutrients to the liver for synthetic activities. In a series of studies we quantified the fate of carbon and nitrogen from $\mathrm{L}-\left[\mathrm{U}-{ }^{14} \mathrm{C}\right]$ glutamine, with or without glucose, in enterocytes from cows in different stages of lactation.

Ammonia production accounted for $53 \%$ and $49 \%$ of the nitrogen from glutamine metabolized to glutamate in enterocytes from early-lactation (EL) and mid-late-lactation (MLL) cows, respectively. In addition, the recovery of glutamine nitrogen taken up by enterocytes was $21 \%$ in alanine and $18 \%$ in glutamate for both $E L$ and $M L L$ and 10 and $9 \%$ in aspartate for EL and MLL cows, respectively. Concomitant addition of glucose and glutamine increased the rate of alanine formation by $54 \%$ vs $93 \%$ and decreased aspartate formation by $18 \%$ vs $40 \%$ in EL and MLL cows, respectively. Glucose probably decreased the formation of aspartate from glutamine by increasing citrate production from glucose-derived acetyl-CoA and glutamine-derived oxaloacetate thereby decreasing the availability of oxaloacetate for transamination. In the absence of glucose, it was calculated that $45 \%$ of glutamate produced in enterocytes from EL cows would need to be converted to pyruvate probably via oxaloacetate by phosphoenolpyruvate carboxykinase and pyruvate kinase and/or via malate by malic enzyme, with pyruvate being converted in part to alanine and lactate. The suggestion of such a pathway of glutamine carbons ending up in pyruvate is equivocal. However, the 54 and $100 \%$ increases in the yield of alanine by inclusion of glucose in enterocytes from EL and MLL cows also suggest that the carbons of alanine may be provided either by the degradation of glutamate to pyruvate and/or by glycolytic pyruvate. In enterocytes from EL and MLL cows, 24 and $20 \%$ of metabolized glutamate carbons appeared in ${ }^{14} \mathrm{CO}_{2}$ However, the presence of glucose decreased the percentage of metabolized glutamate carbons that appeared in ${ }^{14} \mathrm{CO}_{2}$ by $63 \%$ and $67 \%$ in enterocytes from EL and MLL cows, respectively.

We conclude that glutamine plays a role in the provision of not only carbon for oxidation purposes but also nitrogen for biosynthetic processes in the lactating cow.
Products (nmol)

Glutamate

Ammonia

Aspartate

Alanine

$$
4 \mathrm{mM}\left[\mathrm{U}-{ }^{14} \mathrm{C}\right] \mathrm{gln}
$$

4.1

2.9

12.4

8.0

2.3

1.5

5.0

3.4

$$
\left[\mathrm{U}-{ }^{14} \mathrm{C}\right] \mathrm{gln}+6 \mathrm{mM} \text { glucose }
$$

4.5

2.2

10.7

7.2

1.9

0.9

7.7 